\title{
Unified approach for universal quantum gates in a coupled superconducting two-qubit system with fixed always-on coupling
}

\author{
Zhongyuan Zhou, ${ }^{1,2}$ Shih-I Chu, ${ }^{1}$ and Siyuan $\mathrm{Han}^{2}$ \\ ${ }^{1}$ Department of Chemistry, University of Kansas, Lawrence, Kansas 66045, USA \\ ${ }^{2}$ Department of Physics and Astronomy, University of Kansas, Lawrence, Kansas 66045, USA
}

(Received 27 October 2005; published 30 March 2006)

\begin{abstract}
We demonstrate that in a coupled two-qubit system any single-qubit gate can be decomposed into $|0\rangle$-controlled and $|1\rangle$-controlled two-qubit gates which can be implemented by manipulations analogous to that used for a controlled NOT (СNOT) gate. Based on this we present a unified approach to implement universal single-qubit and two-qubit gates in a coupled two-qubit system with fixed always-on coupling. This approach requires neither supplementary circuit or additional physical qubits to control the coupling nor extra hardware to adjust the energy level structure. The feasibility of this approach is demonstrated by numerical simulation of single-qubit gates and creation of two-qubit Bell states in rf-driven inductively coupled two superconducting quantum interference device flux qubits with realistic device parameters and constant always-on coupling.
\end{abstract}

DOI: 10.1103/PhysRevB.73.104521

PACS number(s): 03.67.Lx, 03.67.Pp, 85.25.Dq

During the past decade, a variety of physical qubits have been explored for possible implementation of quantum gates. Of those solid-state qubits based on superconducting devices have attracted much attention because of their advantages of large-scale integration and easy connection to conventional electronic circuits. ${ }^{1-15}$ Superconducting single-qubit gates ${ }^{4-11}$ and two-qubit gates ${ }^{12-15}$ have been demonstrated recently.

However, building a practical quantum computer requires the simultaneous operation of a large number of multiqubit gates in a coupled multiqubit system. It has been proved theoretically that any type of multiqubit gate can be decomposed into a set of universal single-qubit gates and a twoqubit gate, such as the controlled-NOT (CNOT) gate. ${ }^{16,17}$ Thus it is imperative to implement the universal single-qubit and two-qubit gates in a multiqubit system with the minimum resource and maximum efficiency. ${ }^{18}$

Implementing universal single-qubit gates and two-qubit gates in coupled multiqubit systems can be achieved by turning off and on the coupling between qubits. ${ }^{18-21}$ In these schemes, supplementary circuits were required to control interqubit coupling. However, rapid switching of the coupling results in two serious problems. The first one is gate errors caused by population propagation between qubits. Because the computational states of the single-qubit gates are not a subset of the eigenstates of the two-qubit gates the populations of the computational states propagate from one qubit to another when the coupling is changed, resulting in additional gate errors. The second one is additional decoherence introduced by the supplementary circuits. ${ }^{22,23}$ This is one of the biggest obstacles for quantum computing with solid-state qubits, particularly in coupled multiqubit systems. ${ }^{2}$ In addition, the use of supplementary circuits also significantly increases the complexity of fabrication and manipulation of the coupled qubits.

To circumvent these problems, a couple of alternative schemes, such as those with untunable coupling ${ }^{22}$ and always-on interaction, ${ }^{24}$ have been proposed. In the first scheme, each logic qubit is encoded by extra physical qubits and coupling between the encoded qubits is constant but can be turned off and on effectively by putting the qubits in and driving them out of the interaction free subspace. In the second scheme, the coupling is always on but the transition energies of the qubits are tuned individually or collectively. These schemes can overcome the problem of undesired population propagation but still suffer from those caused by the supplementary circuits needed to move the encoded qubits and tune the transition energies. Recently, a protocol for universal gates in coupled superconducting qubits with fixed coupling was proposed, ${ }^{25}$ in which the qubits have detuned and fixed Larmor frequencies during gate operation. The advantage of this scheme is that it requires minimal extra hardware and control lines. However, to perform two-qubit gates one needs a sequence of seven pulses and each pulse's amplitude and phase must be controlled dynamically with high precision. This level of complexity make it quite difficult to realize in practice.

In this paper, we present a unified approach to implement universal single-qubit and two-qubit gates in a coupled twoqubit system with fixed always-on coupling. This approach can be used to construct scalable quantum information processing with simpler and fewer hardware resources when it is combined with other schemes. ${ }^{18-22,24}$ In this approach, each single-qubit gate is realized via two controlled two-qubit gates which we call $|0\rangle$-controlled and $|1\rangle$-controlled twoqubit gates, respectively. The $|0\rangle(|1\rangle)$-controlled gate implements a desired gate in one of the two coupled qubits when the other is in $|0\rangle(|1\rangle)$. Notice that the conventional CNOT gate is a special case of the $|1\rangle$-controlled two-qubit gate. The approach needs no extra circuits or physical qubits in addition to what would be required to perform CNOT gate. Moreover, since the computational states of the single-qubit gates are a subset of those of the two-qubit gates the gate errors due to population propagation are completely eliminated. The effectiveness of the approach is demonstrated by numerically simulating the single-qubit gates and creating the Bell states in a unit of inductively coupled two superconducting quantum interference device (SQUID) flux qubits with realistic device parameters and constant always on coupling. 


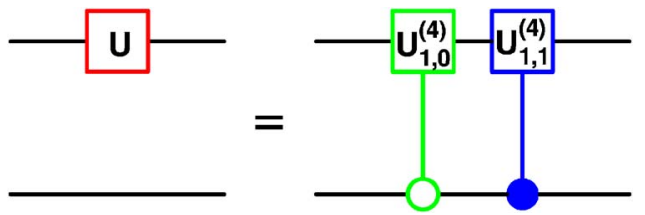

FIG. 1. (Color online) Single-qubit operation $U$ on $\mathcal{Q} 1$ and its equivalent operations by two controlled two-qubit gates $U_{1,0}^{(4)}$ and $U_{1,1}^{(4)}$ in a coupled two-qubit system. The $|0\rangle$-controlled gate and $|1\rangle$-controlled gate are denoted by the open and closed circles for the state of $\mathcal{Q} 2$ being $|0\rangle$ and $|1\rangle$, respectively.

Let us consider a basic unit consisting of two coupled qubits, labeled, respectively, as qubit $1(\mathcal{Q} 1)$ and qubit 2 $(\mathcal{Q} 2)$ for convenience. An eigenstate of the coupled qubits is denoted by $\mid n)=|i j\rangle$, which can be well approximated by the product of an eigenstate of $\mathcal{Q} 1,|i\rangle$, and that of $\mathcal{Q} 2,|j\rangle$, $\mid n) \equiv|i j\rangle=|i\rangle|j\rangle$, for weak interqubit coupling. The computational states of the coupled qubits are $\mid 1)=|00\rangle, \mid 2)=|01\rangle$, $\mid 3)=|10\rangle$, and $|4\rangle=|11\rangle$, which correspond to the eigenstates for $i, j=0,1$ and $n=1,2,3,4$, respectively. In general, the result of an operation on $\mathcal{Q} 1$ depends on the state of $\mathcal{Q} 2$ since $\mathcal{Q} 1$ is coupled to and hence influenced by $\mathcal{Q} 2$. As a consequence, any unitary operation of the coupled qubits, $U$, will act on states in the four-dimensional (4D) Hilbert space spanned by $\mid n)$. For $U$ being a single-qubit operation on $\mathcal{Q} 1$ one wants the state of $\mathcal{Q} 1$ to evolve according to $U$ independent of the state of $\mathcal{Q} 2$ which is left unchanged. Thus all single-qubit gates on $\mathcal{Q} 1$ can be represented by $U_{1}^{(4)}=U_{1}^{(2)} \otimes I_{2}^{(2)}$, where the $4 \times 4$ unitary matrix $U_{1}^{(4)}$ and the $2 \times 2$ unitary matrix $U_{1}^{(2)}$ are the representations of $U$ in the Hilbert space of the coupled qubits and in the subspace of $\mathcal{Q} 1$ while the $2 \times 2$ unitary matrix $I_{2}^{(2)}$ is the representation of the identity operation in the subspace of $\mathcal{Q} 2$. If the matrix elements of $U_{1}^{(2)}$ are $u_{\mu \nu}(\mu, \nu=1,2)$ the operation can be decomposed into two operations as

$$
\begin{aligned}
U_{1}^{(4)} & =\left(\begin{array}{cccc}
1 & 0 & 0 & 0 \\
0 & u_{11} & 0 & u_{12} \\
0 & 0 & 1 & 0 \\
0 & u_{21} & 0 & u_{22}
\end{array}\right)\left(\begin{array}{cccc}
u_{11} & 0 & u_{12} & 0 \\
0 & 1 & 0 & 0 \\
u_{21} & 0 & u_{22} & 0 \\
0 & 0 & 0 & 1
\end{array}\right) \\
& =U_{1,1}^{(4)} U_{1,0}^{(4)} \\
& =U_{1,0}^{(4)} U_{1,1}^{(4)},
\end{aligned}
$$

where the $4 \times 4$ unitary matrices $U_{1,0}^{(4)}$ and $U_{1,1}^{(4)}$ represent $|0\rangle$ controlled and $|1\rangle$-controlled operations on $\mathcal{Q} 1$ in the $4 \mathrm{D}$ Hilbert space of the coupled qubits since $U_{1,0}^{(4)}$ only involves the states $|00\rangle$ and $|10\rangle$ and $U_{1,1}^{(4)}$ only involves $|01\rangle$ and $|11\rangle$. Namely, when the state of $\mathcal{Q} 2$ is $|0\rangle(|1\rangle), U_{1,0}^{(4)}\left(U_{1,1}^{(4)}\right)$ implements the desired single-qubit operation $U$ while $U_{1,1}^{(4)}\left(U_{1,0}^{(4)}\right)$ does nothing. Equation (1) shows that for the coupled qubits any single-qubit gate on one qubit can be obtained by a joint application of the $|0\rangle$-controlled and $|1\rangle$-controlled two-qubit gates regardless of the state of the other qubit. This property is universal and is shown schematically in Fig. 1, where the single-qubit operation $U$ and its equivalent operation via two controlled two-qubit gates $U_{1,0}^{(4)}$ and $U_{1,1}^{(4)}$ are illustrated.

The most obvious advantage of implementing single-qubit gates this way is that the single-qubit gates can be realized using essentially the same set of operations as those for controlled two-qubit gates in the coupled qubits without additional hardware resources. Without loss of generality, let us consider a rf-driven coupled two-qubit system with sufficiently different energy-level spacings $\Delta E_{13}, \Delta E_{24}, \Delta E_{12}$, and $\Delta E_{34}$, which is readily realizable with engineered solid-state qubits, where $\Delta E_{n n^{\prime}}$ is the level spacing between the states $\mid n)$ and $\left.\mid n^{\prime}\right)$. To implement, for example, a single-qubit NOT gate on $\mathcal{Q} 1$, we apply two $\pi$ pulses to the $\mathcal{Q} 1$ : the first one is resonant with $\Delta E_{13}$ and the second with $\Delta E_{24}$. Because both pulses are largely detuned from $\Delta E_{12}$ and $\Delta E_{34}$, they do not induce unintended population transfer when the fields are sufficiently weak. If the state of $\mathcal{Q} 2$ is $|0\rangle(|1\rangle)$, the first (second) pulse accomplishes the desired transformation. Therefore when the two microwave pulses are applied to $\mathcal{Q} 1$, either sequentially or simultaneously, the NOT gate is accomplished and the gate time is essentially the same as that of a stand-alone $\mathcal{Q} 1$ if both pulses are applied simultaneously.

To implement a two-qubit gate such as CNOT in coupled qubits, we use $\mathcal{Q} 1$ and $\mathcal{Q} 2$, respectively, as the control qubit and target qubit and apply a $\pi$ pulse resonant with $\Delta E_{34}$ to $\mathcal{Q} 2 .{ }^{26}$ In this case, the state of $\mathcal{Q} 2$ flips if and only if the state of $\mathcal{Q} 1$ is $|1\rangle$. Since the energy level structure for the singlequbit gates and the CNOT gate could be the same the universal single-qubit gates and CNOT gate can be implemented in the same fashion without adjusting interqubit coupling as long as $\mathcal{Q} 1$ and $\mathcal{Q} 2$ can be addressed individually by microwave pulses which is straightforward to realize, through control/microwave lines of each qubit, with engineered solid-state qubits in general and with rf SQUID flux qubits in particular.

For the sake of concreteness, we demonstrate how to realize the single-qubit and two-qubit gates in rf-driven two SQUID flux qubits with constant always-on coupling. The coupled flux qubits comprise two SQUIDs coupled inductively through their mutual inductance $M$. Each SQUID consists of a superconducting loop of inductance $L$ interrupted by a Josephson tunnel junction characterized by its critical current $I_{c}$ and shunt capacitance $C .{ }^{27} \mathrm{~A}$ flux-biased SQUID with total magnetic flux $\Phi$ enclosed in the loop is analogous to a "flux" particle of mass $m=C \Phi_{0}^{2}$ moving in a one-dimensional potential, where $\Phi_{0}=h / 2 e$ is the flux quantum. For simplicity, we assume that the two SQUIDs are identical. In this case, $C_{i}=C, L_{i}=L$, and $I_{c i}=I_{c}$ for $i=1,2$. The Hamiltonian of the coupled qubits is ${ }^{26,28}$ $H\left(x_{1}, x_{2}\right)=H_{0}\left(x_{1}\right)+H_{0}\left(x_{2}\right)+H_{12}\left(x_{1}, x_{2}\right)$, where $H_{0}\left(x_{i}\right)$ is the Hamiltonian of the $i$ th single qubit given by $H_{0}\left(x_{i}\right)$ $=p_{i}^{2} / 2 m+m \omega_{L C}^{2}\left(x_{i}-x_{e i}\right)^{2} / 2-E_{J} \cos \left(2 \pi x_{i}\right)$ and $H_{12}$ is the interaction between the SQUIDs given by $H_{12}$ $=m \omega_{L C}^{2} \kappa\left(x_{1}-x_{e 1}\right)\left(x_{2}-x_{e 2}\right)$. Here, $x_{i}=\Phi_{i} / \Phi_{0}$ is the canonical coordinate of the $i$ th "flux" particle and $p_{i}=-i \hbar \partial / \partial x_{i}$ is the canonical momentum conjugate to $x_{i}, x_{e i}=\Phi_{e i} / \Phi_{0}$ is the normalized external flux bias of the $i$ th qubit, $E_{J}$ $=m \omega_{L C}^{2} \beta_{L} / 4 \pi^{2}$ is the Josephson coupling energy, $\beta_{L}$ $=2 \pi L I_{C} / \Phi_{0}$ is the potential shape parameter, $\omega_{L C}=1 / \sqrt{L C}$ is the characteristic frequency of the SQUID, and $\kappa=M / L$ is 
the coupling strength. The coupled SQUID qubits are a multilevel system. The eigenenergies $E_{n}$ and eigenstates $\left.\mid n\right)$ are obtained by numerically solving the eigenvalue equation of $H\left(x_{1}, x_{2}\right)$ using the two-dimensional Fourier-grid Hamiltonian method. ${ }^{29}$ When $x_{e 1}$ and $x_{e 2} \sim 0.5$ the coupled SQUID qubits have four wells in the potential energy surface. ${ }^{26}$ The four computational states are chosen to be the lowest eigenstate of each well.

To realize single-qubit and two-qubit gates in the coupled SQUID qubits, we apply microwave pulses $x_{C}$ and $x_{T}$ to $\mathcal{Q} 1$ and $\mathcal{Q} 2$, respectively. The interaction of the coupled qubits and pulses is $V\left(x_{1}, x_{2}, t\right)=d_{1}\left(x_{1}-x_{e 1}\right)+d_{2}\left(x_{2}-x_{e 2}\right)$ $+d_{12}$ with $d_{1}=m \omega_{L C}^{2}\left(x_{C}+\kappa x_{T}\right), \quad d_{2}=m \omega_{L C}^{2}\left(x_{T}+\kappa x_{C}\right)$, and $d_{12}=m \omega_{L C}^{2}\left(x_{C}^{2}+x_{T}^{2}+2 \kappa x_{C} x_{T}\right) / 2$. The time-dependent wave functions of the coupled SQUID qubits, $\psi\left(x_{1}, x_{2}, t\right)$, are governed by the time-dependent Schrödinger equation $i \hbar \partial \psi / \partial t=\left[H\left(x_{1}, x_{2}\right)+V\left(x_{1}, x_{2}, t\right)\right] \psi$. To solve this equation we expand $\psi$ in terms of the first 20 eigenstates of the coupled qubits: $\left.\psi=\Sigma_{n} c_{n}(t) \mid n\right)$. The expansion coefficients $c_{n}(\tau)$ are calculated by numerically solving the matrix equation $i \partial c_{n}(\tau) / \partial \tau=\Sigma_{n^{\prime}} H_{n n^{\prime}}^{R}(\tau) c_{n^{\prime}}(\tau)$ using the split-operator method, ${ }^{30}$ where $\tau=\omega_{L C} t$ is the dimensionless time and $H_{n n^{\prime}}^{R}$ $=\left[E_{n} \delta_{n n^{\prime}}+\left(n|V| n^{\prime}\right)\right] / \hbar \omega_{L C}$ is the reduced Hamiltonian matrix element. The probability of being in the state $\mid n)$ is thus $\left|c_{n}(\tau)\right|^{2}$. For single-qubit gates two resonant pulses, $x_{C 1}$ $=x_{C 10} \cos \left(\omega_{C 1} t\right)$ with $\omega_{C 1}=\Delta E_{13} / \hbar$ and $x_{C 2}=x_{C 20} \cos \left(\omega_{C 2} t\right)$ with $\omega_{C 2}=\Delta E_{24} / \hbar$, are applied simultaneously to the $\mathcal{Q} 1$ so that $x_{C}=x_{C 1}+x_{C 2}$. For controlled two-qubit gates such as the CNOT gate one resonant pulse $x_{T}=x_{T 0} \cos \left(\omega_{T} t\right)$ with $\omega_{T}$ $=\Delta E_{34} / \hbar$ is applied to the $\mathcal{Q} 2$. To minimize the possible intrinsic gate errors caused by leakage to unintended states, we select the parameters of the coupled SQUID qubits using the independent transition approach. ${ }^{31}$ For SQUIDs with $L$ $=100 \mathrm{pH}, C=40 \mathrm{fF}$, and $\beta_{L}=1.2$ one set of the better working parameters is $x_{e 1}=0.499, x_{e 2}=0.4998$, and $\kappa=2.5 \times 10^{-4}$, which will be used in the calculation.

Most of the single-qubit gates, such as the NOT gate and Hadamard gate, can be described by rotations of the state vector on the Bloch sphere. ${ }^{16}$ Thus we demonstrate how to realize an arbitrary single-qubit rotation of an angle $\theta$ about an axis perpendicular to the $z$ axis, $R^{(2)}(\theta)$, in the coupled SQUID qubits. Based on Eq. (1), the rotation $R_{1}^{(4)}(\theta)$ on $\mathcal{Q} 1$ is accomplished via two controlled two-qubit rotations $R_{1,0}^{(4)}(\theta)$ and $R_{1,1}^{(4)}(\theta)$ as shown in Fig. 2(a). They are realized by applying two microwave pulses $x_{C 1}$ and $x_{C 2}$ to $\mathcal{Q} 1$ simultaneously. The amplitudes and frequencies of $x_{C 1}$ and $x_{C 2}$ are $x_{C 10}=5 \times 10^{-5}, \omega_{C 1}=0.239 \omega_{L C}=2 \pi \times 19.0 \mathrm{GHz}$, $x_{C 20}=5.14 \times 10^{-5}$, and $\omega_{C 2}=0.259 \omega_{L C}=2 \pi \times 20.6 \mathrm{GHz}$. In Figs. 2(b) and 2(c), we plot the rotation angle $\theta$ and the populations of the states $|00\rangle$ and $|10\rangle$ (populations of the other states remain essentially at zero) as a function of pulse width when the initial state of the coupled qubits is $|00\rangle$. It is shown in Fig. 2(b) that the rotation angle $\theta$ is essentially a linear function of the pulse width. This indicates that the state of $\mathcal{Q} 1$ undergoes Rabi oscillations for which the phase angle $\Omega \tau$ is a linear function of pulse width, where $\Omega$ is the Rabi frequency. It is also shown in Fig. 2(c) that from the initial state $|00\rangle$ the coupled qubits evolve into the state

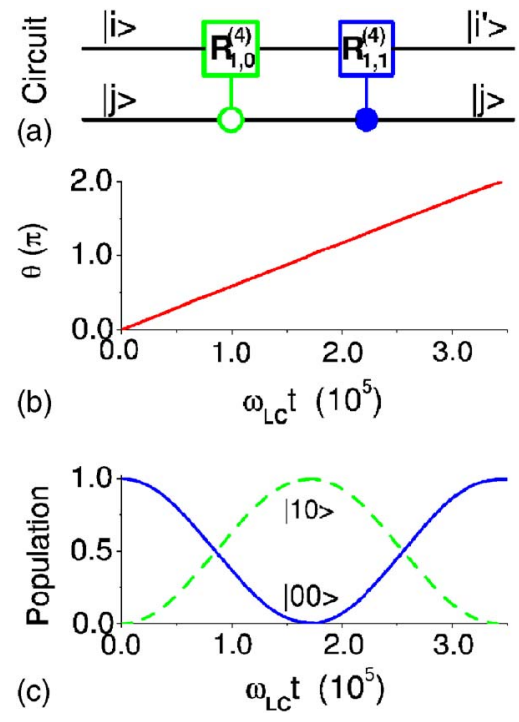

FIG. 2. (Color online) Arbitrary single-qubit rotation about an axis perpendicular to $z$ axis in the coupled SQUID flux qubits. (a) Quantum circuit: the state $|i j\rangle$ evolves to the state $\left|i^{\prime} j\right\rangle=R_{1}^{(4)}(\theta)|i j\rangle$ $=\left[R_{1}^{(4)}(\theta)|i\rangle\right]|j\rangle$ after the two controlled two-qubit rotations. (b) The rotation angle $\theta$ vs pulse width. (c) Populations of the states $|00\rangle$ and $|10\rangle$ vs pulse width.

$(|00\rangle+|10\rangle) / \sqrt{2}$ after two $\pi / 2$ pulses and into the state $|10\rangle$ after two $\pi$ pulses. We have also computed the rotation angles and populations for the coupled qubits with the initial states $|01\rangle,|10\rangle$, and $|11\rangle$ using the same pulse sequence. In each case, the state is transformed from $|i j\rangle$ to $R_{1}^{(4)}(\theta)|i j\rangle$ $=\left[R_{1}^{(4)}(\theta)|i\rangle\right]|j\rangle$ for $i, j=0,1$ with $\theta=\Omega \tau$ as expected.

The quality of a gate can be quantified by normalized gate fidelity $F \equiv \overline{\operatorname{Tr}}\left[\rho_{P} \rho_{I}\right] / \operatorname{Tr}\left[\rho_{I} \rho_{I}\right]$, where $\rho_{P}$ and $\rho_{I}$ are the physical and ideal density matrices after gate operation and the overline denotes averaging over all possible initial states. ${ }^{31,32}$ The fidelities of the single-qubit gates in Fig. 2(c) are quite high. For example, $F=0.9998$ for the Hadamard gate and $F=0.9963$ for the NOT gate, which demonstrate the effectiveness of the approach.

Entanglement is one of the most profound characteristics of quantum systems and a cornerstone of quantum information processing and communication. ${ }^{16}$ The maximally entangled two-qubit states are referred to as the Bell states. To create the Bell states from a state $|i j\rangle$, a Hadamard gate on $\mathcal{Q} 1, H_{1}^{(4)}$, which is decomposed into two controlled two-qubit Hadamard gates $H_{1,0}^{(4)}$ and $H_{1,1}^{(4)}$, and a CNOT gate are commonly used [see Fig. 3(a)]. The two controlled two-qubit Hadamard gates are implemented by applying two $\pi / 2$ pulses $x_{C 1}$ and $x_{C 2}$ to $\mathcal{Q} 1$ and the following CNOT gate is implemented by applying a $\pi$ pulse $x_{T}$ to $\mathcal{Q} 2$, as shown in Fig. 3(b). The amplitudes and frequencies of $x_{C 1}$ and $x_{C 2}$ are the same as those used in Fig. 2 and those of $x_{T}$ are $x_{T 0}=5 \times 10^{-5}$ and $\omega_{T}=0.0592 \omega_{L C}=2 \pi \times 4.7 \mathrm{GHz}$. In Fig. $3(\mathrm{c})$, we plot the population evolution of the computational states when the initial state is $|00\rangle$. It is shown clearly that the coupled qubits evolve first into a product state $(|00\rangle+|10\rangle) / \sqrt{2}$ from the initial state $|00\rangle$ after the $\pi / 2$ pulses and then into a Bell state $(|00\rangle+|11\rangle) / \sqrt{2}$ after the subse- 

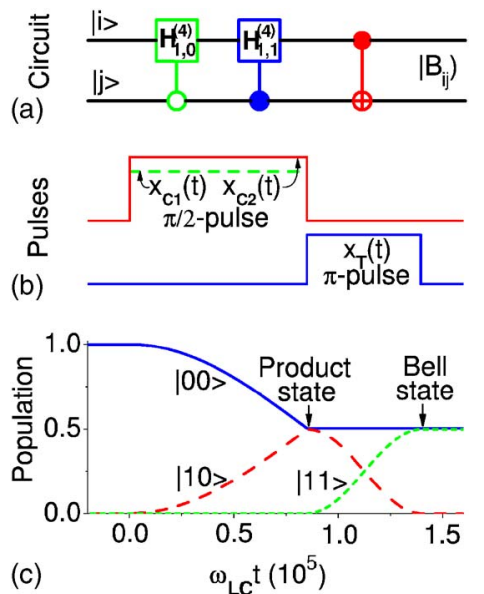

FIG. 3. (Color online) Creation of the Bell states in the coupled SQUID flux qubits. (a) Quantum circuit: the state $|i j\rangle$ evolves into an entangled state $\left(B_{i j}\right)$ after two controlled two-qubit Hadamard gates and one CNOT gate. (b) Microwave pulses: two $\pi / 2$ pulses $x_{C 1}$ and $x_{C 2}$ are applied to the $\mathcal{Q} 1$ first and then a $\pi$ pulse $x_{T}$ is applied to the $\mathcal{Q} 2$. (c) Population evolution: the coupled qubits evolve into a product state $(|00\rangle+|10\rangle) / \sqrt{2}$ from the initial state $|00\rangle$ after the first two $\pi / 2$ pulses and then into a Bell state $(|00\rangle+|11\rangle) / \sqrt{2}$ from the product state after the second $\pi$ pulse.

quent $\pi$ pulse. We have also calculated the population evolution for the coupled qubits being initially in $|01\rangle,|10\rangle$, and $|11\rangle$, respectively. The final state in each case is also one of the expected Bell states. During this process, $F=0.9998$ for the Hadamard gate and $F=0.9999$ for the CNOT gate.

The unified approach is efficient only for a basic unit containing a few coupled qubits because for a unit with $N$ qubits it needs $2^{N-1}$ pulses and requires the computational energy levels to be unequally spaced. However, the scalable quantum information processing for a system with a large number of qubits can be achieved by a hybrid architecture $(\mathrm{HA})^{25}$ in which adjacent basic units are linked by adjustable couplers. ${ }^{18-22,24}$ The HA can significantly reduce the complexity of the circuit compared to the conventional scheme (CS) which uses a qubit as a basic unit. For the sake of concreteness let us consider a generic 1D array of an even number of $N$ qubits with the nearest neighbor coupling. ${ }^{22,24}$ In this architecture the CS needs $N-1$ adjustable couplers, while the HA only needs $N / 2-1$ adjustable couplers if each basic unit contains, for example, two qubits with fixed always-on coupling. Compared to the CS the HA saves about $50 \%$ in the couplers for large $N$. Further let us consider an rf-driven $N$-qubit gate which comprises a Hadamard gate on each single qubit followed by a CNOT gate on the nearest neighbors from left to right. If the initial state is $|00 \cdots 0\rangle$ the system will be in an entangled state after this gate. To implement this gate the CS needs $2 N-2$ microwave pulses and $2 N-2$ operations on the couplers, while the HA needs $3 N-6$ microwave pulses and N-2 operations on the couplers. Although the HA requires about $N$ pulses more than the CS it saves about $N$ operations on the couplers which are usually adiabatic and thus much slower.

In summary, we showed that in a coupled two-qubit system any single-qubit gate can be decomposed into two controlled two-qubit gates which can be implemented with manipulations analogous to that used for the CNOT gate. Based on this universal property of single-qubit gates we present a general approach to implement the universal single-qubit and two-qubit gates in the same coupled two-qubit system with fixed always-on coupling. This approach is demonstrated by using a unit of two SQUID flux qubits with realistic device parameters and constant always-on coupling. Compared to other methods ${ }^{18-22,24,25}$ our approach for a unit with two coupled qubits has the following characteristics and advantages: (1) The approach is universal as long as each qubit can be locally addressed; (2) no extra hardware resources required; (3) no additional decoherence from the hardware added to control interqubit coupling; (4) gate errors induced by the population propagation from one qubit to another is completely eliminated; and (5) the architecture for both hardware (circuits) and software (pulse sequence) is much simplified. For a system with $N$ coupled qubits the scalable quantum information processing can be achieved by the hybrid architecture.

This work was supported in part by the NSF (DMR0325551) and by AFOSR, NSA, and ARDA through DURINT Grant No. F49620-01-1-0439.
${ }^{1}$ A. J. Leggett, Science 296, 861 (2002).

${ }^{2}$ J. Clarke, Science 299, 1850 (2003).

${ }^{3}$ G. Blatter, Nature (London) 421, 796 (2003).

${ }^{4}$ Y. Nakamura, Y. A. Pashkin, and J. S. Tsai, Nature (London) 398, 786 (1999).

${ }^{5}$ D. Vion, A. Aassime, A. Cottet, P. Joyez, H. Pothier, C. Urbina, D. Esteve, and M. H. Devoret, Science 296, 886 (2002).

${ }^{6}$ Y. Yu, S. Han, X. Chu, S.-I. Chu, and Z. Wang, Science 296, 889 (2002).

${ }^{7}$ S. Han, Y. Yu, X. Chu, S.-I. Chu, and Z. Wang, Science 293, 1457 (2001).

${ }^{8}$ J. M. Martinis, S. Nam, J. Aumentado, and C. Urbina, Phys. Rev. Lett. 89, 117901 (2002).
${ }^{9}$ J. R. Friedman, V. Patel, W. Chen, S. K. Tolpygo, and J. E. Lukens, Nature (London) 406, 43 (2000).

${ }^{10}$ C. H. van der Wal, A. C. J. ter Haar, F. K. Wilhelm, R. N. Schouten, C. J. P. M. Harmans, T. P. Orlando, S. Lloyd, and J. E. Mooij, Science 290, 773 (2000).

${ }^{11}$ I. Chiorescu, Y. Nakamura, C. J. P. M. Harmans, and J. E. Mooij, Science 299, 1869 (2003).

${ }^{12}$ Y. A. Pashkin, T. Yamamoto, O. Astafiev, Y. Nakamura, D. V. Averin, and J. S. Tsai, Nature (London) 421, 823 (2003).

${ }^{13}$ T. Yamamoto, Y. A. Pashkin, O. Astafiev, Y. Nakamura, and J. S. Tsai, Nature (London) 425, 941 (2003).

${ }^{14}$ A. J. Berkley, H. Xu, R. C. Ramos, M. A. Gubrud, F. W. Strauch, P. R. Johnson, J. R. Anderson, A. J. Dragt, C. J. Lobb, and F. C. 
Wellstood, Science 300, 1548 (2003).

${ }^{15}$ R. McDermott, R. W. Simmonds, M. Steffen, K. B. Cooper, K. Cicak, K. D. Osborn, S. Oh, D. P. Pappas, and J. M. Martinis, Science 307, 1299 (2005).

${ }^{16}$ M. A. Nielsen and I. L. Chuang, Quantum Computation and Quantum Information (Cambridge University Press, Cambridge, England, 2000).

${ }^{17}$ A. Barenco, C. H. Bennett, R. Cleve, D. P. DiVincenzo, N. Margolus, P. Shor, T. Sleator, J. A. Smolin, and H. Weinfurter, Phys. Rev. A 52, 3457 (1995).

${ }^{18}$ B. L. T. Plourde, J. Zhang, K. B. Whaley, F. K. Wilhelm, T. L. Robertson, T. Hime, S. Linzen, P. A. Reichardt, C.-E. Wu, and J. Clarke, Phys. Rev. B 70, 140501(R) (2004).

${ }^{19}$ J. Q. You, Y. Nakamura, and F. Nori, Phys. Rev. B 71, 024532 (2005).

${ }^{20}$ D. V. Averin and C. Bruder, Phys. Rev. Lett. 91, 057003 (2003).

${ }^{21}$ A. Blais, A. Maassen van den Brink, and A. M. Zagoskin, Phys. Rev. Lett. 90, 127901 (2003).

${ }^{22}$ X. Zhou, Z.-W. Zhou, G.-C. Guo, and M. J. Feldman, Phys. Rev.
Lett. 89, 197903 (2002).

${ }^{23}$ S. C. Benjamin, Phys. Rev. Lett. 88, 017904 (2002).

${ }^{24}$ S. C. Benjamin and S. Bose, Phys. Rev. Lett. 90, 247901 (2003).

${ }^{25}$ C. Rigetti, A. Blais, and M. Devoret, Phys. Rev. Lett. 94, 240502 (2005).

${ }^{26}$ Z. Zhou, S.-I. Chu, and S. Han, IEEE Trans. Appl. Supercond. 15, 833 (2005).

${ }^{27}$ V. V. Danilov, K. Likharev, and A. B. Zorin, IEEE Trans. Magn. 19, 572 (1983).

${ }^{28}$ J. E. Mooij, T. P. Orlando, L. Levitov, L. Tian, C. H. van der Wal, and S. Lloyd, Science 285, 1036 (1999).

${ }^{29}$ S.-I. Chu, Chem. Phys. Lett. 167, 155 (1990).

${ }^{30}$ M. R. Hermann and J. A. Fleck, Jr., Phys. Rev. A 38, 6000 (1988).

${ }^{31}$ Z. Zhou, S.-I. Chu, and S. Han, Phys. Rev. Lett. 95, 120501 (2005).

${ }^{32}$ X. Li, Y. Wu, D. Steel, D. Gammon, T. H. Stievater, D. S. Katzer, D. Park, C. Piermarocchi, and L. J. Sham, Science 301, 809 (2003). 